\title{
Disfunção sexual em endometriose: uma revisão sistemática
}

\section{Sexual dysfunction in endometriosis: a systematic review}

\author{
Alessandra B. Trovó de Marquii ${ }^{1}$, Maria Paula C. Silva², Gabriela R. F. Irie ${ }^{3}$
}

\begin{abstract}
RESUMO
Este estudo teve como objetivo avaliar a função sexual das pacientes com endometriose, bem como a participação de fatores de risco. Foi realizada uma pesquisa bibliográfica no PubMed, em março de 2014, empregando os seguintes descritores: quality of sex life and endometriosis, sexual life and endometriosis, female sexual dysfunction and endometriosis e sexual functioning and endometriosis. Foram encontrados 152 artigos e de acordo com os critérios de inclusão e exclusão previamente estabelecidos, 20 foram selecionados. Treze estudos investigaram a contribuição do tratamento na disfunção sexual feminina. Ansiedade e depressão são fatores que também influenciam a função sexual, mas foram investigadas em apenas três estudos. As pacientes com endometriose exibem comprometimento da função sexual e, portanto, a investigação sobre sua vida sexual deve ser averiguada, tendo em vista os prejuízos causados pela disfunção na qualidade de vida das mulheres.
\end{abstract}

Palavras-chave: Disfunção Sexual Fisiológica. Endometriose. Fatores de Risco. Sexualidade. Saúde da Mulher.

\begin{abstract}
This study aimed to evaluate the sexual function of patients with endometriosis, as well as the involvement of risk factors. A literature search was performed in PubMed, in March 2014, using the following key words: quality of sex life and endometriosis, sexual life and endometriosis, female sexual dysfunction and endometriosis e sexual functioning and endometriosis.152 articles were found, according to the inclusion and exclusion criteria previously established, 20 were selected. Thirteen studies investigated the contribution of treatment in female sexual dysfunction. Anxiety and depression are factors that also influence sexual function, but has been investigated in only three studies. Patients with endometriosis exhibit impaired sexual function and, therefore, research into your sex life should be investigated, in view of the damage caused by dysfunction in the quality of life of women.
\end{abstract}

Keywords: Sexual Dysfunction, Physiological. Endometriosis. Risk Factors. Sexuality. Women's Health.

1. Professora Associada da Disciplina de Genética da Universidade Federal do Triângulo Mineiro (UFTM), Uberaba/MG.

2. Acadêmica do Curso de Graduação em Enfermagem da UFTM, Uberaba/MG.

3. Acadêmica do Curso de Graduação em Biomedicina da UFTM, Uberaba/MG.
Correspondencia Alessandra Bernadete Trovó de Marqui UFTM, Instituto de Ciências Biológicas e Naturais/ICBN, Departamento de Patologia, Genética e Evolução

Disciplina de Genética Campus I - Praça Manoel Terra, № 330 CEP: 38015-050 - Uberaba-MG.

Artigo recebido em 27/06/2014 Aprovado para publicação em 12/02/2015 


\section{Introdução}

A Organização Mundial de Saúde (OMS) referencia a sexualidade humana como sendo um dos indicadores de qualidade de vida (QV). Nesse sentido, uma vida sexual satisfatória é parte integrante da saúde global do ser humano e do bem-estar individual. A resposta sexual saudável é baseada no modelo trifásico: desejo, excitação e orgasmo, o qual é a base de classificação das disfunções sexuais femininas (DSF). A disfunção sexual (DS) é definida como uma situação na qual o indivíduo não consegue concretizar uma relação sexual ou em que esta seja insatisfatória para si e/ou para o seu companheiro. É considerada problema de saúde pública que afeta significativamente a vida das mulheres e de seus parceiros. ${ }^{1,2}$

A DSF é definida como qualquer alteração ou dor em uma ou mais etapas do ciclo da resposta sexual. Nesse sentido, as disfunções sexuais são classificadas em quatro categorias: transtornos ou distúrbios de desejo sexual (desejo sexual hipoativo e aversão sexual), transtornos ou distúrbios da excitação sexual, transtornos ou distúrbios do orgasmo e transtornos ou distúrbios sexuais dolorosos (onde se inclui a dispareunia e o vaginismo). A dor associada à relação sexual gera sofrimento, angústias ou dificuldade interpessoal tornando a mulher incapaz de participar da relação sexual como desejaria. . $^{1,3,4}$

A dispareunia é definida como dor genital que ocorre antes, durante ou após o coito, o que pode levar a mulher a evitar o sexo. Ela é um dos sintomas da endometriose, uma condição ginecológica crônica que afeta mulheres em idade reprodutiva. Os sintomas clínicos da doença compreendem dor pélvica crônica (DPC), dismenorréia, disquezia, disúria, infertilidade e dispareunia. ${ }^{5} \mathrm{~A}$ frequência de dispareunia em mulheres com endometriose variou de $54,7 \%$ a $86 \%$. $^{6-}$ ${ }^{10}$ Com o objetivo de avaliar o impacto da dispareunia na vida e relacionamento das mulheres com endometriose, Denny \& Mann ${ }^{6}$ realizaram um estudo qualitativo com 30 mulheres com a doença. Os resultados mostraram que, a experiência da dor limitou a atividade sexual para a maioria das mulheres investigadas e isso resultou em baixa auto-estima com consequente efeito negativo na relação com o parceiro. Nessa direção, apesar da dispareunia ser um problema sexual comum em pacientes com endometriose, o impacto na sexualidade, identidade feminina e relacionamento dessas pacientes tem sido raramente alvo de investigação. ${ }^{11}$ Um estudo sobre sexualidade em 26 pacientes com endometriose, através de uma abordagem psicossomática e sexológica, mostrou que a resposta clínica da dor foi satisfatória em todas as pacientes na vigência do tratamento e isso promoveu uma melhoria na sexualidade em 21 pacientes, incluindo um melhor relacionamento com os parceiros. ${ }^{12}$

O diagnóstico das DSF é clínico e baseado em uma anamnese detalhada e exame físico efetuado por um ginecologista. Esse profissional deve ter amplo conhecimento da fisiologia e patologia sexual para caracterizar distúrbios somáticos que interfiram na resposta sexual da mulher. O critério diagnóstico inclui o período mínimo de seis meses de sintomatologia e na consulta médica, seria importante a presença do casal para que seja excluída algum distúrbio relacionado ao parceiro. Ainda, caso fatores emocionais estejam associados, a paciente com DSF deve ser encaminhada à psicoterapia. ${ }^{13}$

Apesar do ginecologista ter um papel fundamental no diagnóstico das queixas sexuais relatadas pelas mulheres, há uma falha no ensino da sexualidade nos cursos médicos do Brasil. Duas pesquisas independentes com estudantes de Medicina dos estados do Piauí e São Paulo mostraram uma visão da sexualidade centrada nos aspectos biológicos e patológicos, com pequeno destaque para a construção social da sexualidade. ${ }^{14,15}$ Ainda, uma delas mostrou um significativo percentual de respostas equivocadas sobre sexualidade feminina. ${ }^{14}$ Ambos os estudos mostraram que a formação médica recebida foi limitada para responder às diretrizes que norteiam a assistência em saúde sexual e reprodutiva das políticas públicas de saúde, não abrangendo a sexualidade em seus múltiplos aspectos. ${ }^{14,15}$

Dois fatores interferem no diagnóstico das DSF. Um deles é que uma pequena parcela das mulheres relata suas dificuldades sexuais ao ginecologista por inibição, vergonha ou frustração ou o próprio médico não investiga por constrangimento ou desconhecimento da resposta sexual humana. ${ }^{1}$ Ainda, caso o tema sexualidade seja abordado, as queixas das mulheres são baseadas no seu autorrelato. O ideal seria a utilização de instrumentos de auto-avaliação que contemplem todas as dimensões do funcionamento sexual. ${ }^{16}$

A aplicação de questionários tais como o SHOW-Q (Sexual Health Outcomes in Women Questionnaire), o SAQ (Sexual Activity Questionnaire), o GRISS (Golombok Rust Inventory of Sexual Satisfaction), o FSFI (Female Sexual Function Index), o MFSQ (McCoy Female Sexuality Questionnaire), 
o DSFI/GSSI (Derogatis Sexual Functioning Inventory/Global Sexual Satisfaction Index) auxiliaria o ginecologista no diagnóstico das DSF. No entanto, tais questionários são administrados apenas em pesquisas clínicas. Tais instrumentos permitiriam o conhecimento da extensão dos problemas sexuais o que possibilitaria o oferecimento de abordagens terapêuticas mais eficazes já que a DS é um dos principais determinantes de redução da QV.

Ainda, vale destacar que a endometriose é uma doença crônica e essa exerce influência na QV sexual. Somado a isso, a dispareunia constitui um distúrbio sexual doloroso e um dos sintomas mais frequentes da endometriose. Diante do exposto, este estudo tem como objetivo avaliar a função sexual em pacientes com endometriose, bem como a participação de fatores de risco.

\section{Metodologia}

Foi realizada uma pesquisa bibliográfica no PubMed, em março de 2014, sem limite de período, empregando os seguintes descritores: quality of sex life and endometriosis, sexual life and endometriosis, female sexual dysfunction and endometriosis e sexual functioning and endometriosis. O PubMed foi a base de dados escolhida por ser amplamente utilizada em pesquisas na área da saúde. Os critérios de inclusão foram estarem intimamente relacionados ao tema e no idioma inglês. Foram excluídos artigos do tipo revisão, carta ao editor, relato de caso, aqueles sem relação direta com o tema e publicados em outro idioma que não fosse o inglês. Os artigos repetidos foram contados apenas uma vez. A seleção dos artigos foi feita por dois autores, de forma independente, obedecendo aos critérios de inclusão e exclusão. Nos casos em que houve discordância entre eles, a opinião de terceiro autor foi empregada. O Quadro I apresenta o resultado da busca no PubMed por estudos que abordaram função sexual em endometriose.

\section{Resultados}

Na presente revisão da literatura, foram inclusos 20 artigos científicos que abordaram função sexual em mulheres com endometriose. Os principais resultados desses estudos constam apresentados no Quadro II.

\section{Discussão}

A análise da função sexual feminina (FSF) é influenciada por vários fatores, dentre eles: ansiedade, depressão, escolaridade, idade, renda, alterações hormonais fisiológicas como gestação, amamentação e menopausa, doenças crônicas, infertilidade, medicação (anticoncepcionais orais e antidepressivos), abuso sexual, raiva, estresse, baixa auto-estima, fatores culturais e religiosos, aspectos do relacionamento conjugal, cirurgia, paridade e doenças neurológica, endócrina, vascular e uroginecológica. ${ }^{35-40} \mathrm{Em}$ endometriose, os seguintes fatores merecem destaque: infertilidade, medicação, cirurgia, ansiedade e depressão. Os anticoncepcionais orais e a cirurgia constituem opções terapêuticas para o tratamento da dor pélvica e infertilidade associada à endometriose. ${ }^{41}$ Cerca de 30 a $50 \%$ das mulheres com endometriose são inférteis ${ }^{42}$ e a infertilidade está associada com DS. ${ }^{43}$ Dos vinte estudos apresentados no Quadro II, apenas um deles avaliou oito fatores associados a DS (idade, renda, nível educacional, estado civil, infertilidade, intensidade da dor pélvica, endometriose

\section{Quadro I: Resultados da busca eletrônica realizada em 19/03/2014 no PubMed.}

\begin{tabular}{|c|c|c|}
\hline Descritores & $\begin{array}{l}\text { № de artigos } \\
\text { encontrados }\end{array}$ & $\begin{array}{c}\text { № de artigos } \\
\text { selecionados para leitura }\end{array}$ \\
\hline Quality of sex life and endometriosis & 16 & 7 \\
\hline Sexual life and endometriosis & 58 & 9 \\
\hline Female sexual dysfunction and endometriosis & 63 & 3 \\
\hline Sexual functioning and endometriosis & 15 & 1 \\
\hline Total & 152 & 20 \\
\hline
\end{tabular}




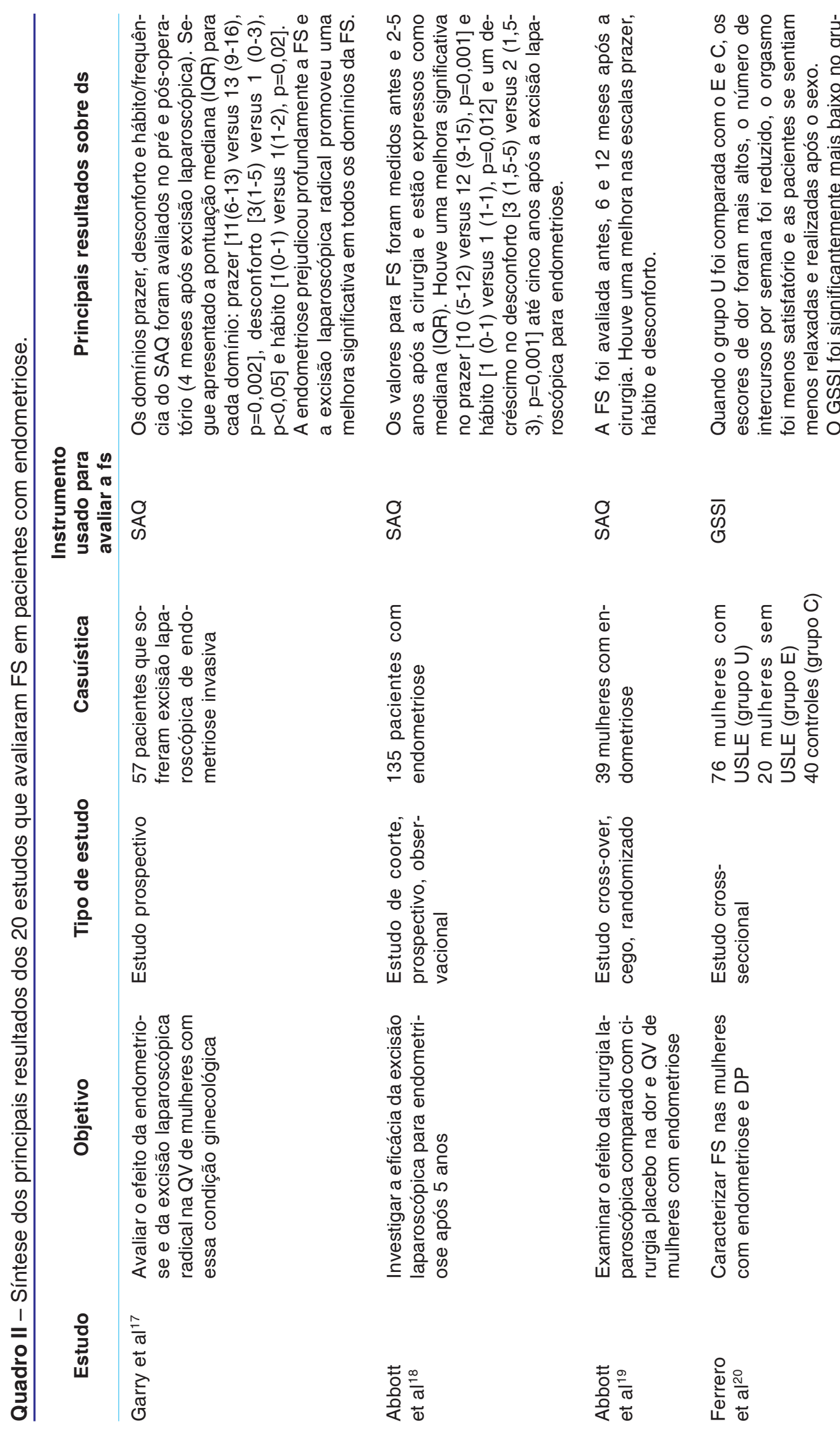




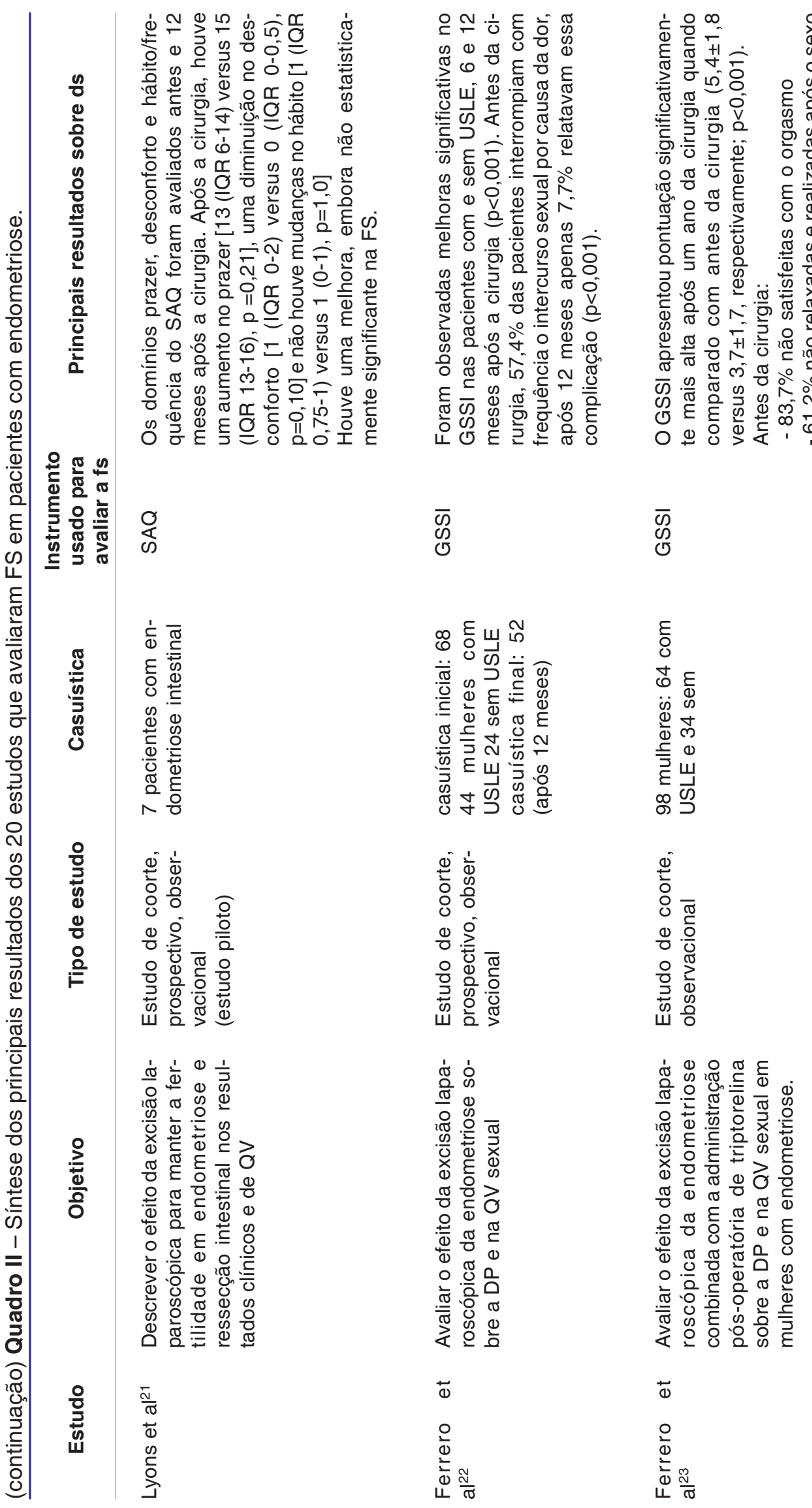




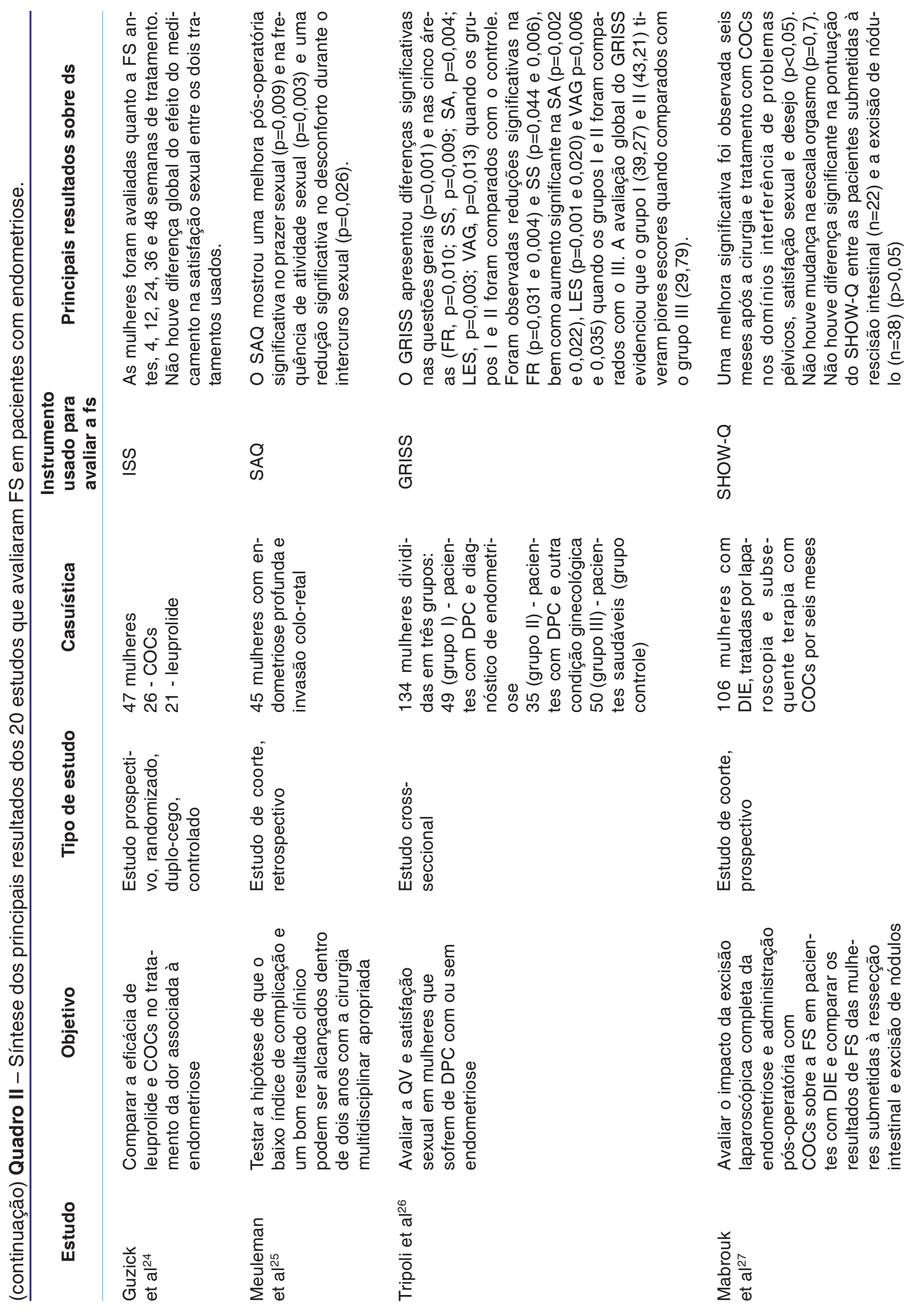




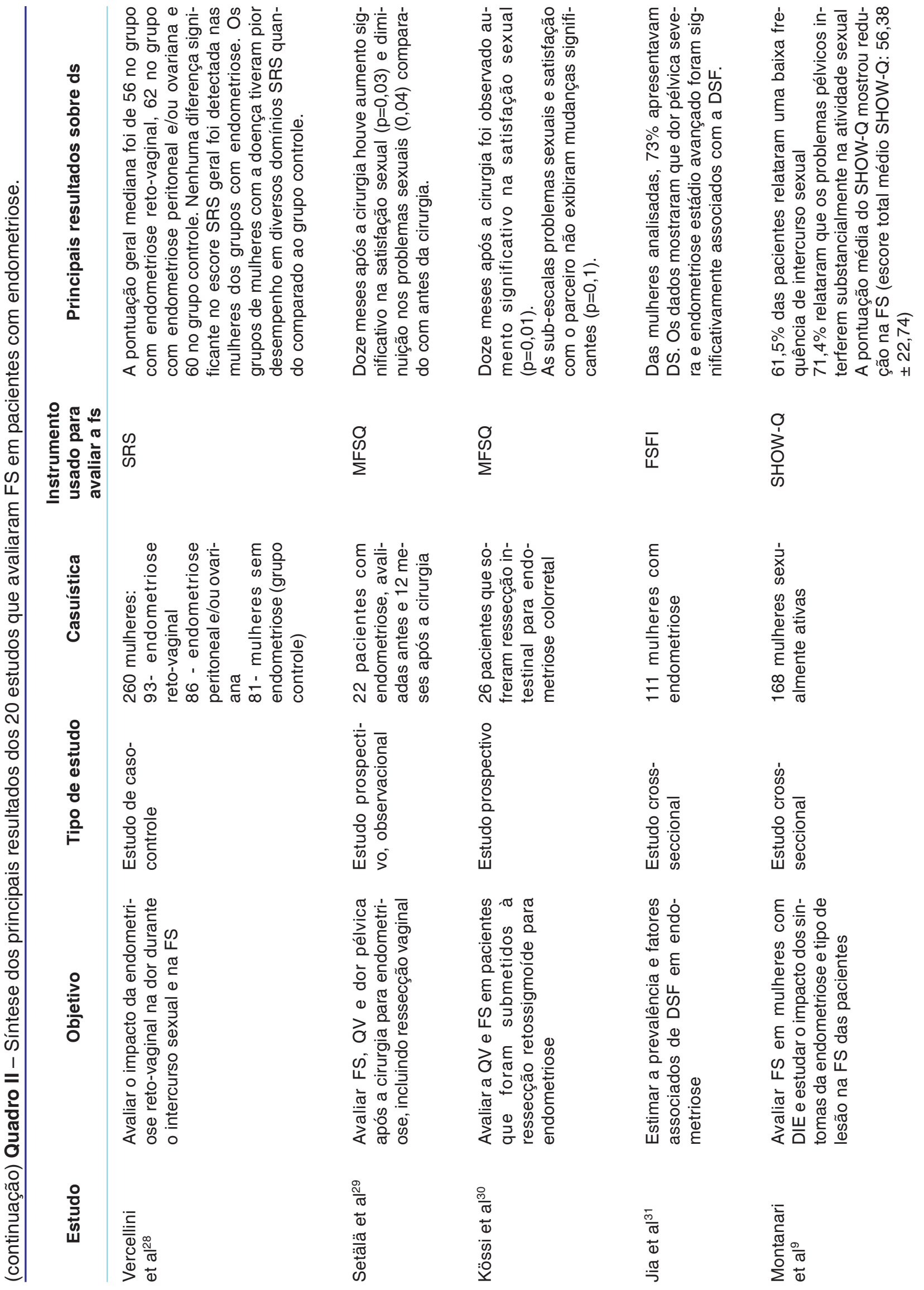




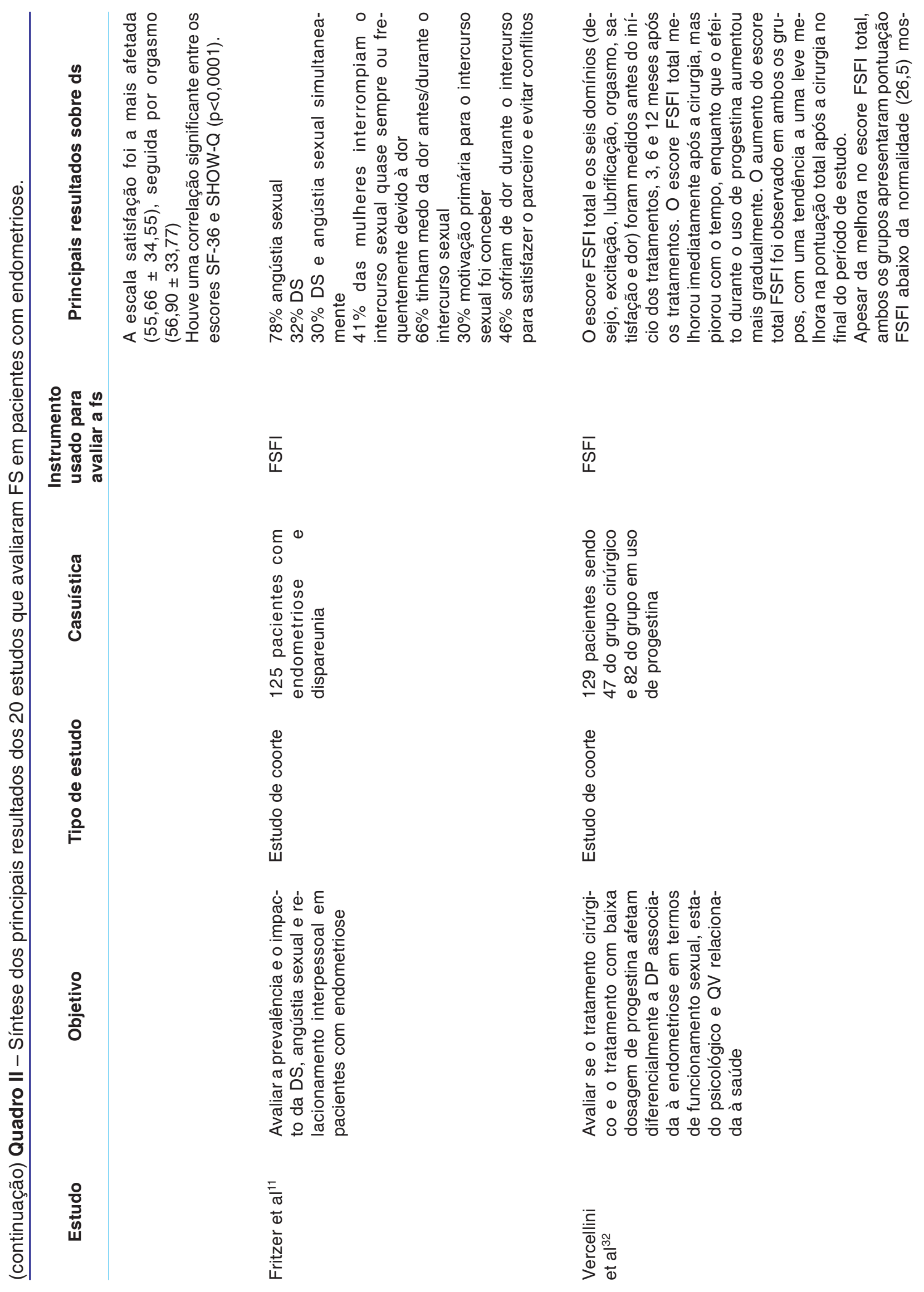




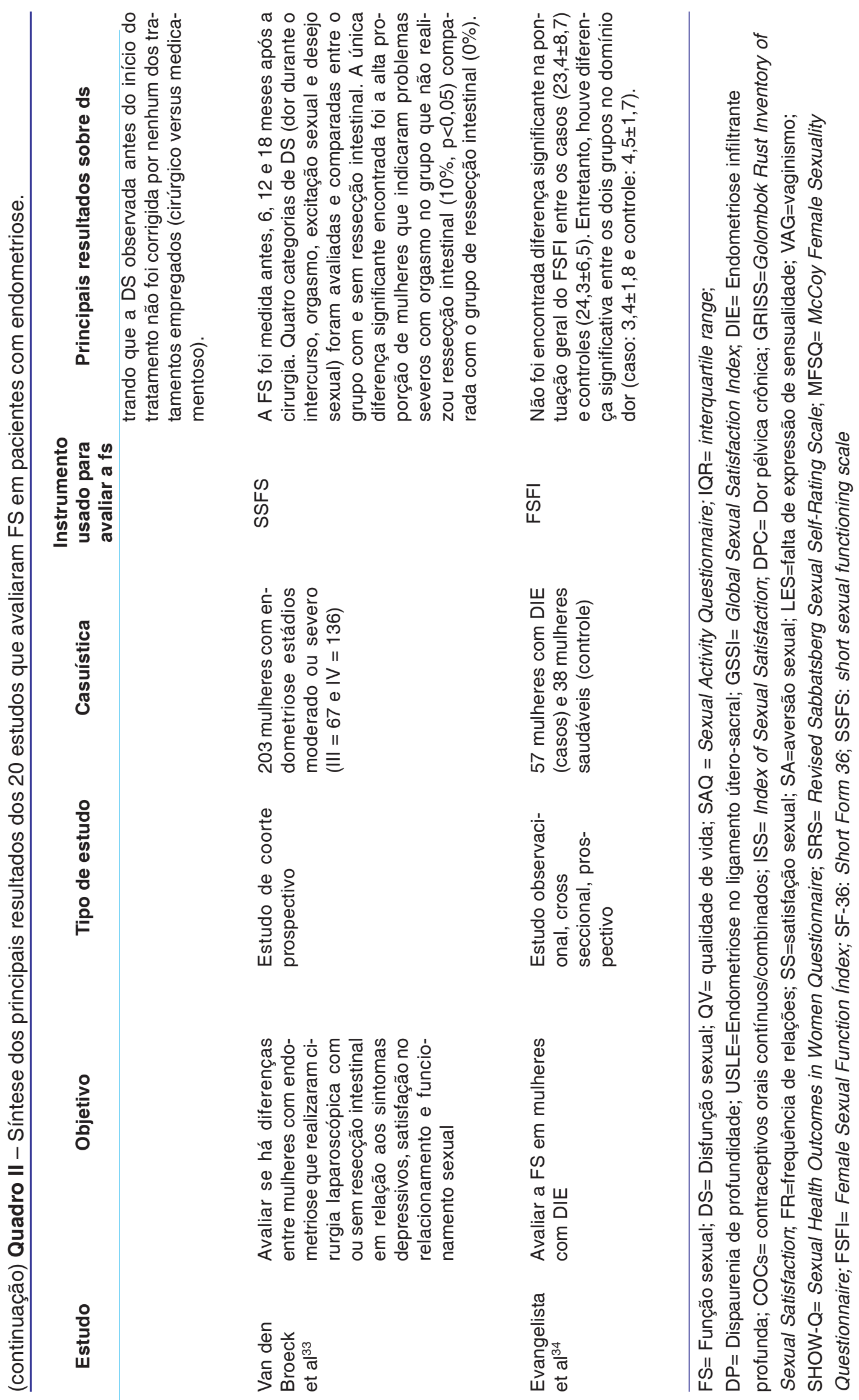


infiltrante profunda e estadiamentos da endometriose). ${ }^{31}$ Treze estudos investigaram a contribuição do tratamento na DSF sendo que nove avaliaram o efeito da cirurgia, ${ }^{17,18,19,21,22,25,29,30,33}$ um o papel do medicamento ${ }^{24}$ e três a combinação de ambos os tratamentos. ${ }^{23,27,32}$ A análise da contribuição do medicamento para a função sexual ficou restrita. Ferrero et al. ${ }^{23}$ sugerem que sejam realizados tratamento clínico ou cirúrgico isolado para se determinar a contribuição de cada um na função sexual. Os sete estudos restantes não analisaram o efeito do tratamento na DSF. ${ }^{9}, 11,20,26,28,31,34$ Ainda, a avaliação da função sexual foi o objeto principal de estudo em 14 artigos científicos $9,11,20,22,23,26-34$ e em seis deles, foi investigada secundariamente à QV e dor. ${ }^{17,18,19,21,24,25}$

Um achado que despertou a atenção foi que apenas quatro ${ }^{20,26,28,34}$ dos sete estudos que não investigaram o efeito do tratamento na DSF, utilizaram grupo controle, ou seja, compararam a função sexual nas mulheres com e sem endometriose. Ainda, um desses estudos destacou como limitação a ausência de grupo controle. ${ }^{9}$ A presença do grupo controle permite uma comparação entre indivíduos com a doença com aqueles saudáveis e, portanto, pode-se estabelecer um valor de normalidade para a característica a ser avaliada. É importante o pareamento entre os grupos com características sociodemográficas semelhantes.

A aplicação de instrumentos de auto-avaliação tem se mostrado eficaz na análise da FSF. Os questionários SAQ, FSFI e GSSI foram os mais utilizados, sendo empregados em $60 \%$ dos estudos. Em virtude da ampla heterogeneidade de questionários aplicados para análise da FSF, como apresentado no Quadro II, a comparação dos resultados tornou-se limitada.

Os estudos que empregaram o SAQ ${ }^{17,18,19,21,25}$ mostraram que a cirurgia promoveu um aumento no prazer, uma diminuição no desconforto e em três deles houve também elevação na frequência/hábito de intercurso sexual ${ }^{18,19,25}$. O domínio desconforto do SAQ corresponde à presença de ressecamento vaginal e dor ou desconforto durante a penetração. A diminuição da dor após a cirurgia também foi relatada por Ferrero et al. ${ }^{22}$. Dois outros estudos ${ }^{29,30}$ empregaram o mesmo instrumento e observaram aumento da satisfação sexual e apenas um deles, diminuição dos problemas sexuais. ${ }^{29}$ Essa última categoria avalia lubrificação insuficiente e relações sexuais dolorosas, ou seja, corresponde aos domínios desconforto e dor do SAQ e GSSI, respectivamente.
O instrumento FSFI foi empregado em quatro estudos. ${ }^{11,31,32,34}$ Esse questionário avalia seis domínios: desejo, excitação, lubrificação, orgasmo, satisfação e dor. Todas as pesquisas consideraram que um escore FSFI $\leq 26,5$ indicou disfunção sexual. No estudo de Evangelista et al. ${ }^{34}$ o grupo de estudo e o controle apresentaram escores de 23,4 \pm 8,7 e 24,3 \pm 6,5, respectivamente, ou seja, não houve diferença significativa nos escores FSFI total e ambos exibiram disfunção sexual. Apesar do grupo controle não apresentar nenhuma complicação ginecológica ou outra comorbidade, a explicação para esse achado é indefinida. Segundo os autores, uma possível justificativa é que as pacientes com endometriose, embora apresentassem dispareunia profunda, minimizam esse sintoma devido ao desejo de engravidarem. Isto poderia aumentar os escores individuais de alguns domínios do FSFI, elevando o escore total do grupo controle. Nos outros dois estudos publicados em 2013 os autores não informaram a pontuação geral do FSFI para comparação. ${ }^{11,31} \mathrm{Jia}$ et al..$^{31}$ relataram que a prevalência de DSF em mulheres com endometriose foi $73 \%$, e no estudo de Fritzer et al. ${ }^{11}$, a incidência foi muito mais baixa (32\%). Quatro estudos realizados no Brasil em mulheres saudáveis relataram que a prevalência variou de $35,9 \%$ a $53 \% .44-47$ Outro estudo realizado com 214 mulheres portuguesas em idade reprodutiva (18 e 57 anos) evidenciou que a prevalência de DSF foi ainda mais elevada $(77,2 \%) .{ }^{40}$

$\mathrm{Na}$ ausência de tratamento para endometrio$\mathrm{se}^{9,26}$, as pacientes afetadas relataram um aumento da aversão sexual ocasionando uma diminuição na frequência de relações sexuais com satisfação sexual comprometida devido a dispareunia. Esses dados coincidem com aqueles publicados previamente por Denny \& Mann ${ }^{6}$ que mostraram que $86 \%$ das pacientes relataram dispareunia e dessas $69 \%$ apresentavam dor após o intercurso por algumas horas; 69\% evitavam o sexo devido à dor e $19 \%$ não estavam ativas por causa da dor. Outro estudo mostrou que a dispareunia esteve presente como fator de esquiva sexual em parte da amostra e que somente os graus mais avançados de endometriose comprometeram a sexualidade. ${ }^{48}$ Nesse sentido, a dor constituiu fator limitante para o intercurso sexual e isso destaca a importância do tratamento farmacológico e cirúrgico no controle dessa variável. A dor pode ser tratada por excisão peritoneal dos implantes, nódulos profundos e cistos ovarianos ou induzindo supressão da lesão 
por abolir ovulação e menstruação através da manipulação hormonal com progestinas, contraceptivos orais e agonistas de hormônio liberador de gonadotrofina $(\mathrm{GnRHa})$. Esses fármacos criam um ambiente hipoestrogênico (GnRHa - acetato de nafarelina, acetato de leuprolida, triptorelina), hiperandrogênico (danazol, gestrinona) ou hiperprogestogênico (contraceptivos orais, progestágenos - acetato de noretisterona, dienogest, sistema intrauterino com levonorgestrel/ SIU-LNg, acetato de medroxiprogesterona/DMPA), com supressão da proliferação celular endometrial. ${ }^{41,49}$ $\mathrm{Na}$ escolha do medicamento deve-se considerar os efeitos colaterais e grau de satisfação das pacientes com o tratamento. Em relação ao tratamento cirúrgico para dor associada à endometriose, uma revisão recente conclui que esse parece ser a terapia definitiva para as mulheres com sintomas dolorosos exacerbados. ${ }^{50}$ No entanto está associado a complicações e alta taxa de recorrência. ${ }^{49}$ Uma revisão sistemática da literatura mostrou que a recorrência de dor foi relatada por 45 das 189 mulheres com endometriose profunda e recorrência necessitando de reintervenção ocorreu em 61 de 314 mulheres. A recorrência da endometriose foi relatada em 37 de 267 mulheres. ${ }^{51}$ Ainda em relação a dispareunia e função sexual, há um trabalho do tipo revisão que mostrou que a ressecção cirúrgica diminuiu a intensidade da dor e melhorou a QV sexual das pacientes com endometriose. ${ }^{52}$

Apesar dos sintomas psicológicos estarem associados com DS, dos 20 estudos inclusos nessa revisão, apenas três deles avaliaram ansiedade e/ou depressão e função sexual em pacientes com endometriose. ${ }^{24,32,33}$ A depressão ${ }^{53,54,55}$ e a ansiedade ${ }^{55}$ afetam cerca de $90 \%$ das pacientes com essa doença. A primeira está associada com redução da libido e da autoestima, e consequentemente, leva à diminuição da frequência e do interesse sexual. Ainda, o uso de antidepressivos tais como tricíclicos, inibidores seletivos da recaptação da serotonina e inibidores da monoaminoxidase que auxiliam no tratamento da depressão, intensificam a redução da libido interferindo negativamente na QV sexual da paciente. ${ }^{38,56}$ Com relação à ansiedade, Lucena $\& \mathrm{Abdo}^{39}$ concluem que níveis leve ou moderado de ansiedade podem auxiliar na excitação sexual, enquanto graus mais graves pre- judicam o funcionamento sexual. Uma maneira de minimizar os sintomas de ansiedade e depressão e melhorar a QV sexual das mulheres com endometriose seria o uso da Terapia Comportamental Cognitiva. ${ }^{57,58}$ Essa seria uma opção viável tendo em vista que a medicação com antidepressivos pode provocar DSF. Outros métodos disponíveis para as DSF incluem tratamento medicamentoso e fisioterapêutico. ${ }^{2,3,4}$

Considerando a íntima relação endometriose, depressão, ansiedade e DS, seria conveniente a participação de profissionais da psicologia, fisioterapia e terapia sexual na rotina de atendimento das pacientes com essa doença. Essa equipe poderia oferecer às pacientes estratégias terapêuticas voltadas para o tratamento holístico, tirando o foco do tratamento convencional (cirúrgico e medicamentoso) voltado para DPC e infertilidade. Em suma, a endometriose é uma doença crônica e complexa que necessita de uma abordagem holística e interdisciplinar.

De nosso conhecimento, os artigos sobre prevalência e fatores associados à DSF em endometriose são escassos ${ }^{11,31}$, o que sugere a necessidade de estudos adicionais voltados para essa temática. Um estudo recente em mulheres em idade reprodutiva mostrou que a dispareunia foi o segundo subtipo de DSF mais prevalente $(40,9 \%)$. A aversão sexual foi estatisticamente relacionada com existência de uma história prévia de abuso sexual $(\mathrm{p}=0,001)$ e a ingestão de anticoncepcionais hormonais foi associada a uma diminuição do desejo $(\mathrm{p}=0,003) .{ }^{40}$

\section{Conclustå}

Os estudos apresentados nessa revisão mostraram que as pacientes com endometriose exibem comprometimento da função sexual e o tratamento farmacológico e/ou cirúrgico foi eficaz na melhora dessa variável. Deste modo, o impacto da doença sobre a sexualidade da paciente é significativo.

Sugerimos que, a investigação sobre a vida sexual de pacientes com endometriose deve ser averiguada, ou seja, uma atenção especial deve ser dada às queixas sexuais das pacientes durante a consulta ginecológica, tendo em vista os prejuízos causados pela disfunção sexual na QV das mulheres. 


\section{Referências}

1. Lara LA, Rosa e Silva AC, Romão AP, Junqueira FR. [The assessment and management of female sexual dysfunction]. Rev Bras Ginecol Obstet. 2008;30:312-21.

2. Antonioli RS, Simões D. Abordagem fisioterapêutica nas disfunções sexuais femininas. Rev Neurocienc. 2010;18:26774.

3. Mendonça CR, Silva TM, Arrudai JT, García-Zapata MTA, Amaral WN. Função sexual feminina: aspectos normais e patológicos, prevalência no Brasil, diagnóstico e tratamento. Femina. 2012;40:195-202.

4. Buster JE. Managing female sexual dysfunction. Fertil Steril. 2013;100:905-15

5. Bulun SE. Endometriosis. N Engl J Med. 2009;360:268-79.

6. Denny $\mathrm{E}$, Mann $\mathrm{CH}$. Endometriosis-associated dyspareunia: the impact on women's lives. J Fam Plann Reprod Health Care. 2007;33:189-93.

7. Bellelis P, Dias JA Jr, Podgaec S, Gonzales M, Baracat EC, Abrão MS. Epidemiological and clinical aspects of pelvic endometriosis-a case series. AMB rev Assoc Med Bras. 2010;56:467-71.

8. Fourquet J, Gao X, Zavala D, Orengo JC, Abac S, Ruiz A, et al. Patients' report on how endometriosis affects health, work, and daily life. Fertil Steril. 2010;93:2424-8.

9. Montanari G, Di Donato N, Benfenati A, Giovanardi G, Zannoni $L$, Vicenzi $C$, et al. Women with deep infiltrating endometriosis: sexual satisfaction, desire, orgasm, and pelvic problem interference with sex. J Sex Med. 2013;10:1559-66.

10. Smorgick N, Marsh CA, As-Sanie S, Smith YR, Quint EH. Prevalence of pain syndromes, mood conditions, and asthma in adolescents and young women with endometriosis. $\mathrm{J}$ Pediatr Adolesc Gynecol. 2013;26:171-5.

11. Fritzer N, Haas D, Oppelt P, Renner S, Hornung D, Wölfler M, et al. More than just bad sex: sexual dysfunction and distress in patients with endometriosis. Eur J Obstet Gynecol Reprod Biol. 2013;169:392-6.

12. Montgomery $M$, Pereira DHM. Uma abordagem psicossomática e sexológica da endometriose. Reprod. Clim. 1995;10:129-31.

13. Tozo IM, Lima SMRR, Gonçalves N, Moraes JC, Aoki T. Disfunção sexual feminina: a importância do conhecimento e do diagnóstico pelo ginecologista. Arq Med Hosp Fac Cienc Med Santa Casa São Paulo 2007;52:94-9.

14. Lima MCP, Cerqueira ATAR. Crenças sobre sexualidade entre estudantes de Medicina: uma comparação entre gêneros. Rev Bras Educ Med. 2008;32:49-55.

15. Rufino AC, Madeiro AP, Girão MJBC. O Ensino da Sexualidade nos Cursos Médicos: a Percepção de Estudantes do Piauí. Rev Bras Educ Med. 2013; 37:178-85.

16. Lima AC, Dotto LM, Mamede MV. [Prevalence of sexual dysfunction in primigravidae in Rio Branco, Acre State, Brazil]. Cad Saude Publica. 2013;29:1544-54.

17. Garry R, Clayton R, Hawe J. The effect of endometriosis and its radical laparoscopic excision on quality of life indicators. BJOG. 2000;107:44-54.

18. Abbott JA, Hawe J, Clayton RD, Garry R. The effects and effectiveness of laparoscopic excision of endometriosis: a prospective study with 2-5 year follow-up. Hum Reprod. 2003:18:1922-7.

19. Abbott J, Hawe J, Hunter D, Holmes M, Finn P, Garry R. Laparoscopic excision of endometriosis: a randomized, placebo-controlled trial. Fertil Steril. 2004;82:878-84.
20. Ferrero S, Esposito F, Abbamonte LH, Anserini P, Remorgida V, Ragni N. Quality of sex life in women with endometriosis and deep dyspareunia. Fertil Steril. 2005;83:573-9.

21. Lyons SD, Chew SS, Thomson AJ, Lenart M, Camaris C, Vancaillie TG, et al. Clinical and quality-of-life outcomes after fertility-sparing laparoscopic surgery with bowel resection for severe endometriosis. J Minim Invasive Gynecol. 2006;13:436-41.

22. Ferrero S, Abbamonte LH, Giordano M, Ragni N, Remorgida V. Deep dyspareunia and sex life after laparoscopic excision of endometriosis. Hum Reprod. 2007;22:1142-8.

23. Ferrero S, Abbamonte LH, Parisi M, Ragni N, Remorgida V. Dyspareunia and quality of sex life after laparoscopic excision of endometriosis and postoperative administration of triptorelin. Fertil Steril. 2007;87:227-9.

24. Guzick DS, Huang LS, Broadman BA, Nealon M, Hornstein MD. Randomized trial of leuprolide versus continuous oral contraceptives in the treatment of endometriosis-associated pelvic pain. Fertil Steril. 2011;95:1568-73.

25. Meuleman C, Tomassetti C, D'Hoore A, Buyens A, Van Cleynenbreugel B, Fieuws S, et al. Clinical outcome after CO laser laparoscopic radical excision of endometriosis with colorectal wall invasion combined with laparoscopic segmental bowel resection and reanastomosis. Hum Reprod. 2011;26:2336-43.

26. Tripoli TM, Sato H, Sartori MG, de Araujo FF, Girão MJ, Schor E. Evaluation of quality of life and sexual satisfaction in women suffering from chronic pelvic pain with or without endometriosis. J Sex Med. 2011;8:497-503.

27. Mabrouk M, Montanari G, Di Donato N, Del Forno S, Frascà $C$, Geraci E, et al. What is the impact on sexual function of laparoscopic treatment and subsequent combined oral contraceptive therapy in women with deep infiltrating endometriosis? J Sex Med. 2012;9:770-8.

28. Vercellini P, Somigliana E, Buggio L, Barbara G, Frattaruolo MP, Fedele L. "I can't get no satisfaction": deep dyspareunia and sexual functioning in women with rectovaginal endometriosis. Fertil Steril. 2012;98:1503-11.

29. Setälä M, Härkki P, Matomäki J, Mäkinen J, Kössi J. Sexual functioning, quality of life and pelvic pain 12 months after endometriosis surgery including vaginal resection. Acta Obstet Gynecol Scand. 2012;91:692-8.

30. Kössi J, Setälä M, Mäkinen J, Härkki P, Luostarinen M. Quality of life and sexual function 1 year after laparoscopic rectosigmoid resection for endometriosis. Colorectal Dis. 2013;15:102-8.

31. Jia SZ, Leng JH, Sun PR, Lang JH. Prevalence and associated factors of female sexual dysfunction in women with endometriosis. Obstet Gynecol. 2013;121:601-6.

32. Vercellini P, Frattaruolo MP, Somigliana E, Jones GL, Consonni $D$, Alberico D, et al. Surgical versus low-dose progestin treatment for endometriosis-associated severe deep dyspareunia II: effect on sexual functioning, psychological status and health-related quality of life. Hum Reprod. 2013;28:1221-30.

33. Van den Broeck U, Meuleman C, Tomassetti C, D'Hoore A, Wolthuis A, Van Cleynenbreugel B, et al. Effect of laparoscopic surgery for moderate and severe endometriosis on depression, relationship satisfaction and sexual functioning: comparison of patients with and without bowel resection. Hum Reprod. 2013;28:2389-97.

34. Evangelista A, Dantas T, Zendron C, Soares T, Vaz G, Oliveira MA. Sexual function in patients with deep infiltrating endometriosis. J Sex Med. 2014;11:140-5. 
35. Laumann EO, Paik A, Rosen RC. Sexual dysfunction in the United States: prevalence and predictors. JAMA. 1999;281:537-44.

36. Cayan S, Akbay E, Bozlu M, Canpolat B, Acar D, Ulusoy E. The prevalence of female sexual dysfunction and potential risk factors that may impair sexual function in Turkish women. Urol Int. 2004;72:52-7.

37. Lewis RW, Fugl-Meyer KS, Bosch R, Fugl-Meyer AR, Laumann EO, Lizza E, et al. Epidemiology/risk factors of sexual dysfunction. J Sex Med. 2004;1:35-9.

38. Lucena BB, Abdo CHN. Considerações sobre a disfunção sexual feminina e a depressão. Diagn Tratamento. 2012;17:82-5.

39. Lucena BB, Abdo CHN. O papel da ansiedade na (dis)função sexual. Diagn Tratamento. 2013;18:94-8.

40. Ribeiro B, Magalhães AT, Mota I. Disfunção sexual feminina em idade reprodutiva: prevalência e fatores associados. Rev Port Med Geral Fam. 2013; 29:16-24.

41. Nácul AP, Spritzer PM. [Current aspects on diagnosis and treatment of endometriosis]. Rev Bras Ginecol Obstet. 2010;32:298-307.

42. Bulletti C, Coccia ME, Battistoni S, Borini A. Endometriosis and infertility. J Assist Reprod Genet. 2010;27:441-7.

43. Davari Tanha F, Mohseni M, Ghajarzadeh M. Sexual function in women with primary and secondary infertility in comparison with controls. Int J Impot Res. 2014; 26:132-4.

44. Abdo CHN. Perfil sexual da população brasileira: resultado do estudo do comportamento sexual (ECOS) do Brasileiro. RBM Rev Bras Med. 2002; 59:250-7.

45. Abdo CH, Oliveira WM Jr, Moreira ED Jr, Fittipaldi JA. Prevalence of sexual dysfunctions and correlated conditions in a sample of Brazilian women-results of the Brazilian study on sexual behavior (BSSB). Int J Impot Res. 2004;16:160-6.

46. Ferreira ALCG, Souza AI, Amorim MMR. Prevalência das disfunções sexuais femininas em clínica de planejamento familiar de um hospital escola no Recife, Pernambuco. Rev Bras Saúde Matern Infant. Recife 2007; 7:143-50.

47. Valadares AL, Pinto-Neto AM, Osis MJ, Sousa MH, CostaPaiva L, Conde DM. Prevalence of sexual dysfunction and its associated factors in women aged 40-65 years with 11 years or more of formal education: a population-based household survey. Clinics. (São Paulo) 2008;63:775-82.
48. Pereira HGS, Canella PR. Avaliação da satisfação sexual e fertilidade em mulheres com endometrioses. Femina. 2006; 34:175-82

49. Vercellini $P$, Viganò $P$, Somigliana $E$, Fedele L. Endometriosis: pathogenesis and treatment. Nat Rev Endocrinol. 2014; 10:261-75.

50. Kondo W, Zomer MT, Amaral VF. Tratamento cirúrgico da endometriose baseado em evidências. Femina. 2011;39:143-8.

51. De Cicco C, Corona R, Schonman R, Mailova K, Ussia A, Koninckx P. Bowel resection for deep endometriosis: a systematic review. BJOG. 2011;118:285-91.

52. Fritzer N, Tammaa A, Salzer H, Hudelist G. Dyspareunia and quality of sex life after surgical excision of endometriosis: a systematic review. Eur J Obstet Gynecol Reprod Biol. 2014;173:1-6.

53. Lorençatto C, Vieira MJ, Pinto CL, Petta CA. [Evaluation of the frequency of depression in patients with endometriosis and pelvic pain]. AMB rev Assoc Med Bras. 2002; 48:217-21.

54. Lorençatto C, Petta CA, Navarro MJ, Bahamondes L, Matos A. Depression in women with endometriosis with and without chronic pelvic pain. Acta Obstet Gynecol Scand. 2006; 85:8892.

55. Sepulcri R de P, do Amaral VF. Depressive symptoms, anxiety, and quality of life in women with pelvic endometriosis. Eur J Obstet Gynecol Reprod Biol. 2009; 142:53-6.

56. Lee KU, Lee YM, Nam JM, Lee HK, Kweon YS, Lee CT, et al. Antidepressant-Induced Sexual Dysfunction among Newer Antidepressants in a Naturalistic Setting. Psychiatry Investig. 2010; 7:55-9.

57. ter Kuile MM, Both S, van Lankveld JJ. Cognitive behavioral therapy for sexual dysfunctions in women. Psychiatr Clin N Am. 2010; 33: 595-610.

58. Hou Y, Hu P, Zhang Y, Lu Q, Wang D, Yin L, et al. Cognitive behavioral therapy in combination with systemic family therapy improves mild to moderate postpartum depression. Rev Bras Psiquiatr. 2014; 36:47-52. 